\title{
Focused assessment with sonography for trauma: current perspectives
}

This article was published in the following Dove Press journal:

Open Access Emergency Medicine

26 July 2017

Number of times this article has been viewed

\author{
Sorravit Savatmongkorngul' \\ Sirote Wongwaisayawan ${ }^{2}$ \\ Rathachai Kaewlai \\ 'Department of Emergency Medicine, \\ Ramathibodi Hospital, Mahidol \\ University, Bangkok, Thailand, \\ ${ }^{2}$ Division of Emergency Radiology, \\ Department of Diagnostic and \\ Therapeutic Radiology, Ramathibodi \\ Hospital, Mahidol University, Bangkok, \\ Thailand
}

Correspondence: Rathachai Kaewlai Division of Emergency Radiology, Department of Diagnostic and Therapeutic Radiology, Ramathibodi Hospital, 270 Rama VI Road, Ratchathewi, Bangkok 10400, Thailand

Tel +66 855532753

Fax +6622010338

Email rathachai@gmail.com

\begin{abstract}
Focused assessment with sonography for trauma (FAST) is a part of resuscitation of trauma patients recommended by international panel consensus. The purpose of FAST is to identify free fluid, which necessarily means blood in acute trauma patients. In this article, the authors focused on various aspects of FAST in the emergency department, prehospital care, pediatric setting, training and general pearls/pitfalls. Detailed techniques and interpretation of FAST are beyond the scope of this article.
\end{abstract}

Keywords: FAST, trauma, ultrasound, perspectives

\section{Introduction}

Focused assessment with sonography for trauma (FAST) is a part of resuscitation of trauma patients recommended by international panel consensus and incorporated into the advanced trauma life support (ATLS) course. ${ }^{1,2}$ The purpose of FAST is to identify free fluid, which necessarily means blood in acute trauma patients, in three potential body spaces, namely, pericardial, pleural and peritoneal spaces. The four target areas of scanning include the pericardial view, right upper quadrant (RUQ) view, left upper quadrant (LUQ) view and pelvic view. In this article, the authors focused on various aspects of FAST in the emergency department (ED), prehospital care, pediatric setting, training and general pearls/pitfalls. Detailed techniques and interpretation of FAST are beyond the scope of this article.

\section{FAST as a diagnostic tool in ED}

Diagnostic performance of FAST in the ED depends on several factors, including clinical setting, experience of performers, body habitus of the patient, bowel gas interference and equipment. Despite a number of limitations, FAST still has a good sensitivity and specificity for identification of free fluid in trauma patients (Table 1). . $^{3-8}$

FAST has a major role in triage and guiding the diagnosis and management of trauma patients. Ollerton et al found that the management was changed in $32.8 \%$ of patients after FAST. In addition, diagnostic peritoneal lavage (DPL) has decreased from $9 \%$ to $1 \%$, while CT utilization has decreased from $47 \%$ to $34 \%{ }^{9}$

During resuscitation of blunt abdominal trauma patients, FAST is often the first diagnostic imaging modality for patient evaluation. CT is, generally, used after a positive FAST examination in hemodynamically stable patients to evaluate for organ injury. For patients with unstable hemodynamics, FAST can be quickly performed and its result might inform the surgeons on the potential site of hemorrhage. ${ }^{10-17}$ 
In a retrospective study by Moylan et al, positive FAST in normotensive blunt trauma patients is strongly associated with the need for therapeutic laparotomy with an adjusted odds ratio of 44.6 (95\% CI: $1.77-1124)$. The sensitivity, specificity, positive predictive value (PPV) and negative predictive value (NPV) of FAST are 75.8\%, 97.4\%, 37.3\% and $99.5 \%$, respectively. ${ }^{18}$

Interestingly, the false-negative rate of FAST performed in blunt abdominal trauma patients with high injury severity score (ISS) is higher than that performed in patients with ISS of $<25$. Becker et al showed that the sensitivity of FAST is only $65.1 \%$ among patients with ISS of $>25$ when compared with $86.4 \%$ in those with a lower ISS. Specificity is similar in the two groups. Therefore, physicians must be careful when interpreting FAST of patients with multiple trauma, particularly when the result is negative. ${ }^{19}$

In penetrating abdominal trauma, the reported sensitivity of FAST ranges from $28.1 \%$ to $100 \%$, but the specificity is very high from $94.1 \%$ to $100 \%$. Positive FAST in penetrating abdominal trauma indicates intraperitoneal bleeding and may warrant laparotomy. Nevertheless, local wound exploration, DPL or CT should still be considered in patients with negative FAST. ${ }^{20}$

For the detection of solid organ injuries, FAST is neither sensitive nor specific. Reported sensitivity varies from $38 \%$ to $95.4 \%$. The sensitivity for diagnosis gastrointestinal injury was only $38.5 \%$ but increased to $85.2 \%$ when the scan was repeated in 12-24 hours. ${ }^{21}$

In recent years, an additional scan of bilateral anterior hemithoraces to identify pneumothorax has been added to FAST, and the examination has been renamed as extended
FAST (E-FAST). E-FAST is highly accurate for identification of pneumothorax (Table 2), particularly over portable chest radiography often performed in trauma patients..$^{22,23,24} \mathrm{In}$ addition, many trauma centers utilize ultrasound (US) of the inferior vena cava (IVC) to rapidly assess volume status of patients. ${ }^{25}$

\section{Prehospital and out-of-hospital FAST}

Prehospital management of trauma patients is challenging since history, physical examination and initial resuscitation must be as quick, accurate and reliable as much as possible. FAST may be a good diagnostic test for the evaluation of blunt abdominal trauma patients on the field because intraperitoneal fluid, pericardial fluid and pleural fluid can be demonstrated, perhaps, as quickly and easily as in the hospital setting even when being performed by emergency medical technicians (EMTs). ${ }^{26-29}$

The reported sensitivity, specificity, PPV and NPV of FAST performed by EMT for diagnosis of free intraperitoneal fluid are $61.3 \%, 96.3 \%, 89.1 \%$ and $83.2 \%$, respectively, which is quite comparable with those performed by physicians. ${ }^{27}$

FAST or E-FAST can be performed at the site of trauma or during transportation with minimal examination time. In a prospective observational study by Brun et al, the mean examination time of E-FAST is 3.5 minutes for onsite examinations and 3.9 minutes for examinations during patient transfer. The examinations are considered feasible in $95.4 \%$ and $93.9 \%$ of patients onsite and during transfer, respectively. ${ }^{28}$ Even in aeromedical transportation, FAST or

Table I Diagnostic performance of FAST in the ED

\begin{tabular}{llllll}
\hline Studies & Number of patients & Sensitivity, \% & Specificity, \% & PPV, \% & NPV, \% \\
\hline Boulanger et al $^{3}$ & 460 & 96 & 99.7 & 98.7 & NA \\
Brenchley et al $^{4}$ & 153 & 78 & 99 & 99.2 & NA \\
Hsu et al $^{5}$ & 463 & 78 & 97 & 72.4 & 93 \\
Tayal et al $^{6}$ & 96 & 80.8 & 86.9 & 84 & 81.4 \\
Gaarder et al $^{7}$ & 104 & 62 & 96 & 89 & 83 \\
Verbeek et al $^{8}$ & 120 & 64 & 94 & 84 \\
\hline
\end{tabular}

Abbreviations: ED, emergency department; FAST, focused assessment with sonography for trauma; NA, not applicable; NPV, negative predictive value; PPV, positive predictive value.

Table 2 Diagnostic performance of E-FAST for identification of pneumothorax

\begin{tabular}{lllll}
\hline Studies & Sensitivity, \% & Specificity, \% & PPV, \% & NPV, \% \\
\hline Nandipati et al ${ }^{22}$ & 95 & 99 & 95 & 99 \\
lanniello et al $^{23}$ & 77 & 99.8 & 98.5 & 97 \\
\hline
\end{tabular}

Abbreviations: E-FAST, extended focused assessment with sonography for trauma; NPV, negative predictive value; PPV, positive predictive value. 
E-FAST can help physicians in diagnosis and management of injured patients. ${ }^{29}$ This mode of transportation with helicopter emergency medical service has become more common and is currently the method of choice in some trauma patients. The specificity for diagnosis of hemoperitoneum, pericardial effusion and pneumothorax is high at $94.1 \%, 99.6 \%$ and $99.5 \%$, respectively. Flight nurses, paramedics and pilots can also learn to perform FAST in order to incorporate it into daily practice. ${ }^{30}$

FAST also has a major role during war time as a tool for triage of injured soldiers and a guide for laparotomy. The reported sensitivity, specificity, PPV and NPV of FAST in this setting with therapeutic laparotomy as a goal are $55.6 \%$, $97.8 \%, 86.5 \%$ and $89.5 \%$ respectively. ${ }^{31}$

\section{Pediatric FAST}

In the survey study to evaluate the use of US in pediatric trauma in 2002, only $14 \%$ of respondents used FAST in the ED as compared to $74 \%$ of adult patients. ${ }^{32}$ Nowadays, FAST has become popular among emergency physicians, surgeons and pediatric emergency physicians (PEM) alike. ${ }^{33}$ According to a recent survey, 93\% of PEM staff and PEM fellows utilize FAST in pediatric trauma patients. ${ }^{34}$

Diagnostic performance of FAST in children is somewhat different than that of adults. In a recent meta-analysis of trauma US performed in 3,838 children for identifying free fluid, the sensitivity, specificity, positive likelihood ratio and negative likelihood ratio were $80 \%, 96 \%, 22.9 \%$ and $0.2 \%$, respectively. ${ }^{35}$ In another prospective observational study, the sensitivity of FAST in detecting clinically significant hemoperitoneum and any amount of hemoperitoneum is rather low at $52 \%$ and $20 \%$, respectively. The specificity of FAST is $96 \%$ and $98 \%$, while the accuracy is $93 \%$ and $78 \%$, respectively. ${ }^{36}$

FAST examination in pediatric patients can be highly specific but insensitive to detect free intraperitoneal fluid. However, combining FAST and physical examinations can add value in blunt abdominal trauma when compared with FAST examination alone. In the recent investigation, the combination has sensitivity and specificity of $90 \%$ and $83 \%$. When both FAST and physical examinations are normal, nonoperative management may be safely performed. ${ }^{37}$ In another investigation, combining hemodynamics of the patients together with FAST and CT results can improve accuracy of the evaluation of pediatric blunt abdominal trauma. Sensitivity, NPV and negative likelihood ratio rise to $83.3 \%, 99.1 \%$ and 0.167 , respectively. ${ }^{38}$

\section{Training}

Emergency medicine physicians, surgeons, radiologists and resident trainees usually perform emergency US for diagnostic and therapeutic purpose. Currently, training of FAST and other bedside emergency US to medical students is routine in many medical schools as the skills are considered essential for physicians and are not difficult to learn and practice. ${ }^{39-42}$ Furthermore, training of FAST has extended to allied medical professionals such as specialized nurses and EMT since the tool is useful for patient's triage both in the field of trauma and in the ED. ${ }^{39-45}$

Although training seems to be widespread, there is a lack of an international, standardized training curriculum. In addition, competency-based assessment of FAST during training can be challenging. ${ }^{46}$ Quality of ultrasound imaging and competence has been proposed for use in validation of training in FAST that can help discriminate between novice and expert. ${ }^{30}$

In general, training of FAST composes of two parts: lecture and hands-on session. ${ }^{47}$ There seems to be no difference in posttest scores between using a classroom-based lecture and using a web-based lecture. Both types of lecture can improve participants' scores in posttest examinations. ${ }^{48,49}$ For hands-on part, it can be performed using a human model or a simulator. By using a human model, anatomic landmarks are better demonstrated, and the technique is easier to perform and interpret. However, pathological conditions generally cannot be shown. In a simulator model, real-time images can be reconstructed by a computer with an ability to demonstrate both normal and pathological conditions. The downside is the difficulty in acquiring proper images on the mannequin. In a recent systematic review, $64.5 \%$ of FAST training uses human model in hands-on sessions, while only $14.5 \%$ uses a simulator model. The rest uses animals or cadavers. ${ }^{47-51}$ Despite some differences between the two models, both provide significant improvement in physician's confidence from pretest to posttest. ${ }^{50,51}$ Several data supported hands-on FAST training for several groups of health care providers with supervision from experts. ${ }^{39-45}$

Training of FAST using lectures with hands-on sessions can improve knowledge-based outcome, particularly among medical students. ${ }^{39}$ Data from an US symposium for medical students called Ultrafest consisting of hands-on workshops demonstrate an increase in the score of clinical knowledge from 8.1 to 15.6 (out of 21 possible points) among participants. The rate of acceptable FAST images was also increased from $17 \%$ to $47 \% .{ }^{40}$ In another investigation by Blackstock et al, medical students passing a 4-week formal training 
curriculum had mean scores of cognitive skills (physics and image interpretation) increasing from $66.6 \%$ to $85.7 \%$ and the score for image acquisition up to $86.6 \%{ }^{41}$ Training in multiple phases during medical years, including lectures with hands-on sessions and 1-year supervision, can increase a success rate of completion of FAST examination. The score of FAST interpretation increases from 6.2 to 7.6 (out of 10) among participants in the first phase of lecture with handson sessions. Performance of more than 30 FAST scans with supervision during the later phase of training can double the odds of a successful examination. ${ }^{42}$

Extending FAST training to nurses has been shown to provide a significant improvement in skills of emergency nurses. After a 1-day lecture and hands-on session inclusive of scanning more than 25 FAST examinations, participants could achieve sensitivity, specificity, PPV and NPV of $84.4 \%$, $98.4 \%, 94.2 \%$ and $95.3 \%$, respectively. The overall accuracy of nurse-performed FAST is $95 \%$ for the detection of free fluid. ${ }^{43}$ Inclusion of FAST and other emergency US applications in training of emergency nurses can help increase its utilization and patient care. ${ }^{44}$ This situation is likely to be useful in developing countries with resource-limited EDs.

\section{Pitfalls and limitations}

Similar to many other tests, FAST has technical pitfalls, interpretive pitfalls and limitations. Although the RUQ is the most sensitive view, particularly at the caudal edge of the liver, for free fluid detection, ${ }^{52}$ the LUQ view is usually the most challenging view to perform as compared to others, requiring a longer time and much effort to obtain adequate visualization. A classic teaching is to identify free intraperitoneal fluid within the splenorenal recess in this view. However, fluid in the LUQ is more often present in a nearby left paracolic gutter inferior to the spleen, contradicting the traditional knowledge. Free fluid can also be found in the subphrenic space, located between the spleen and the left hemidiaphragm. Therefore, these spaces should be interrogated in the LUQ view of FAST.$^{53}$ Other technical pitfalls of FAST such as poor image gain, suboptimal depth and backward orientation of the transducer are often reduced with an increasing number of examinations, perhaps reflecting more experience of operators. ${ }^{8}$

Another challenge of performing FAST is to balance between sensitivity and specificity. Both false-positive and falsenegative examinations could lead to unfavorable cascade of tests or unnecessary surgery. ${ }^{19,54,55}$ Physicians should perform FAST with caution to avoid false positivity, such as misinterpretation of the double-line sign (DLS) as free fluid and an elongated left hepatic lobe as a splenic hematoma. The DLS is an echogenic line caused by fascial planes encasing the hypoechoic perinephric fat pad, which may have wedge-shaped and sharp borders in the Morison's pouch. ${ }^{54,55}$ It can be found in patients of all ages, and there is no correlation between the amount of perinephric fat and the patient's body mass index. ${ }^{55}$ Misinterpretation of the DLS as free intraperitoneal fluid can lead to unnecessary workup or management for patients. ${ }^{56}$ Elongated left lobe of the liver and splenic subcapsular hematoma sometimes look alike and can be difficult to differentiate from each other in acute trauma patients. ${ }^{57}$ The left hepatic lobe usually has a left hepatic vein or a left portal vein as an anatomical landmark, which can be seen on both gray scale and color Doppler imaging. Another method to confirm the lesion as a part of the liver is to sweep the transducer back to the right hepatic lobe to confirm the continuation.

FAST has a limited utility in the detection of retroperitoneal fluid and small intraperitoneal fluid. The pelvic view is best used to detect small free intraperitoneal fluid with a median volume of detection at a minimum of $100 \mathrm{~mL} .^{58}$ For the detection of free fluid associated with grade I-II hepatosplenic injury, the sensitivity of FAST ranges from $55.6 \%$ to $80 \%$, but this improves as the severity of injury increases. ${ }^{59}$ Therefore, CT scan should be performed if there is a high index of suspicion of injuries in trauma patients with a negative FAST examination. ${ }^{60}$ Follow-up FAST can be an alternative option if the physicians suspect a low-grade injury.

False-negative FAST may be influenced by the severity of both intra-abdominal and other injuries. Tayal et al and Gaarder et al demonstrated that minor solid organ injuries and severe head injuries were associated with the increase of false-negative FAST - albeit not the patient outcome., ${ }^{6,7}$ Their results are supported by a large retrospective cohort study that showed two independent factors - the presence of severe head injury and severe extremity injury - significantly associated with false-negative FAST examination with odds ratios of 4.90 and 1.86, respectively. Interestingly, abdominal organ injuries are not associated with false-negative FAST examination. There is no association between hospital length of stay, ICU length of stay, therapeutic laparotomy or mortality and false-negative FAST examination. ${ }^{61}$

E-FAST may overlook pneumothorax particularly at the areas around the lung apices and bases, which account for two-thirds of false-negative tests. About $30 \%$ of these pneumothoraces require intervention including drainage. ${ }^{62}$ Interpretation of volume assessment by the US of the IVC may be interfered by endotracheal intubation and several underlying cardiopulmonary conditions, such as pulmonary arterial hypertension, cardiac tamponade, tricuspid regurgitation and chronic obstructive pulmonary disease. ${ }^{25}$ 


\section{Conclusion}

FAST is an extremely useful diagnostic tool in triage and guidance in the diagnosis and management of trauma patients, both in- and out-of-hospital. It is an accepted standard in the ATLS protocol that physicians and specialized personnel caring of trauma patients should be adequately trained. Despite its good diagnostic performance and ease of performance, limitations of FAST exist in pediatric age group and those with high injury severity score. It also has some technical and interpretive challenges that physicians should be aware of.

\section{Disclosure}

The authors report no conflicts of interest in this work.

\section{References}

1. Scalea TM, Rodriguez A, Chiu WC, et al. Focused Assessment with Sonography for Trauma (FAST): results from an international consensus conference. J Trauma. 1999;46(3):466-472.

2. Christie-Large M, Michaelides D, James SL. Focused assessment with sonography for trauma: the FAST scan. Trauma. 2008;10(2):93-101.

3. Boulanger BR, McLellan BA, Brenneman FD, Ochoa J, Kirkpatrick AW. Prospective evidence of the superiority of a sonography-based algorithm in the assessment of blunt abdominal injury. J Trauma. 1999;47(4):632-637.

4. Brenchley J, Walker A, Sloan JP, Hassan TB, Venables H. Evaluation of focussed assessment with sonography in trauma (FAST) by UK emergency physicians. Emerg Med J. 2006;23(6):446-448.

5. Hsu JM, Joseph AP, Tarlinton LJ, Macken L, Blome S. The accuracy of focused assessment with sonography in trauma (FAST) in blunt trauma patients: experience of an Australian major trauma service. Injury. 2007;38(1):71-75

6. Tayal VS, Nielsen A, Jones AE, Thomason MH, Kellam J, Norton HJ. Accuracy of trauma ultrasound in major pelvic injury. J Trauma. 2006;61(6):1453-1457.

7. Gaarder C, Kroepelien CF, Loekke R, Hestnes M, Dormage JB, Naess PA. Ultrasound performed by radiologists-confirming the truth about FAST in trauma. J Trauma. 2009;67(2):323-327; discussion 328-329.

8. Verbeek DO, Zijlstra IA, van der Leij C, Ponsen KJ, van Delden OM, Goslings JC. The utility of FAST for initial abdominal screening of major pelvic fracture patients. World J Surg. 2014;38(7):1719-1725.

9. Ollerton JE, Sugrue M, Balogh Z, D’Amours SK, Giles A, Wyllie P. Prospective study to evaluate the influence of FAST on trauma patient management. J Trauma. 2006;60(4):785-791.

10. Kornezos I, Chatziioannou A, Kokkonouzis I, et al. Findings and limitations of focused ultrasound as a possible screening test in stable adult patients with blunt abdominal trauma: a Greek study. Eur Radiol. 2010;20(1):234-238.

11. Deunk J, Brink M, Dekker HM, et al. Predictors for the selection of patients for abdominal CT after blunt trauma: a proposal for a diagnostic algorithm. Ann Surg. 2010;251(3):512-520.

12. Natarajan B, Gupta PK, Cemaj S, Sorensen M, Hatzoudis GI, Forse RA. FAST scan: is it worth doing in hemodynamically stable blunt trauma patients? Surgery. 2010;148(4):695-700; discussion 700-701.

13. Botelho Filho FM, de Oliveira e Silva RC, Starling SV, Zille DP, Drumond DA. Complementary exams in blunt torso trauma. Perform only radiographs and fast: is it safe? Rev Col Bras Cir. 2015;42(4):220 223. Portuguese.

14. Dammers D, El Moumni M, Hoogland II, Veeger N, Ter Avest E. Should we perform a FAST exam in haemodynamically stable patients presenting after blunt abdominal injury: a retrospective cohort study. Scand J Trauma Resusc Emerg Med. 2017;25(1):1.
15. Cook MR, Holcomb JB, Rahbar MH, Fox EE, Alarcon LH, Bulger EM, et al. An abdominal computed tomography may be safe in selected hypotensive trauma patients with positive focused assessment with sonography in trauma examination. Am J Surg. 2015;209(5):834-840.

16. Helling TS, Wilson J, Augustosky K. The utility of focused abdominal ultrasound in blunt abdominal trauma: a reappraisal. Am J Surg. 2007;194(6):728-732; discussion 732-733.

17. Mishra B, Gupta A, Sagar S, Singhal M, Kumar S. Traumatic cardiac injury: experience from a level-1 trauma centre. Chin J Traumatol. 2016;19(6):333-336.

18. Moylan M, Newgard CD, Ma OJ, Sabbaj A, Rogers T, Douglass R. Association between a positive ED FAST examination and therapeutic laparotomy in normotensive blunt trauma patients. J Emerg Med. 2007;33(3):265-271.

19. Becker A, Lin G, McKenney MG, Marttos A, Schulman CI. Is the FAST exam reliable in severely injured patients? Injury. 2010;41(5):479-483.

20. Quinn AC, Sinert R. What is the utility of the Focused Assessment with Sonography in Trauma (FAST) exam in penetrating torso trauma? Injury. 2011;42(5):482-487.

21. Mohammadi A, Ghasemi-Rad M. Evaluation of gastrointestinal injury in blunt abdominal trauma "FAST is not reliable": the role of repeated ultrasonography. World J Emerg Surg. 2012;7(1):2.

22. Nandipati KC, Allamaneni S, Kakarla R, et al. Extended focused assessment with sonography for trauma (EFAST) in the diagnosis of pneumothorax: experience at a community based level I trauma center. Injury. 2011;42(5):511-514.

23. Ianniello S, Di Giacomo V, Sessa B, Miele V. First-line sonographic diagnosis of pneumothorax in major trauma: accuracy of e-FAST and comparison with multidetector computed tomography. Radiol Med. 2014;119(9):674-680.

24. Wongwaisayawan S, Suwannanon R, Sawatmongkorngul S, Kaewlai R. Emergency thoracic US: the essentials. Radiographics. 2016;36(3): 640-659.

25. Richards JR, McGahan JP. Focused Assessment with Sonography in Trauma (FAST) in 2017: what radiologists can learn. Radiology. 2017;283(1):30-48.

26. Ruesseler M, Kirschning T, Breitkreutz R, Marzi I, Walcher F. Prehospital and emergency department ultrasound in blunt abdominal trauma. Eur J Trauma Emerg Surg. 2009;35(4):341.

27. Kim CH, Shin SD, Song KJ, Park CB. Diagnostic accuracy of focused assessment with sonography for trauma (FAST) examinations performed by emergency medical technicians. Prehosp Emerg Care. 2012;16(3):400-406.

28. Brun PM, Bessereau J, Chenaitia H, et al. Stay and play eFAST or scoop and run eFAST? That is the question! Am J Emerg Med. 2014;32(2):166-170.

29. Mazur SM, Pearce A, Alfred S, Goudie A, Sharley P. The F.A.S.T.E.R. trial. Focused assessment by sonography in trauma during emergency retrieval: a feasibility study. Injury. 2008;39(5):512-518.

30. Mohammad A, Hefny AF, Abu-Zidan FM. Focused Assessment Sonography for Trauma (FAST) training: a systematic review. World J Surg. 2014;38(5):1009-1018.

31. Smith IM, Naumann DN, Marsden ME, Ballard M, Bowley DM. Scanning and war: utility of FAST and CT in the assessment of battlefield abdominal trauma. Ann Surg. 2015;262(2):389-396.

32. Baka AG, Delgado CA, Simon HK. Current use and perceived utility of ultrasound for evaluation of pediatric compared with adult trauma patients. Pediatr Emerg Care. 2002;18(3):163-167.

33. Friedman LM, Tsung JW. Extending the focused assessment with sonography for trauma examination in children. Clin Pediatr Emerg Med. 2011;12(1):2-17.

34. Chamberlain MC, Reid SR, Madhok M. Utilization of emergency ultrasound in pediatric emergency departments. Pediatr Emerg Care. 2011;27(7):628-632.

35. Holmes JF, Gladman A, Chang CH. Performance of abdominal ultrasonography in pediatric blunt trauma patients: a meta-analysis. J Pediatr Surg. 2007;42(9):1588-1594. 
36. Fox JC, Boysen M, Gharahbaghian L, et al. Test characteristics of focused assessment of sonography for trauma for clinically significant abdominal free fluid in pediatric blunt abdominal trauma. Acad Emerg Med. 2011;18(5):477-482.

37. Tummers W, van Schuppen J, Langeveld H, Wilde J, Banderker E, van As A. Role of focused assessment with sonography for trauma as a screening tool for blunt abdominal trauma in young children after high energy trauma. S Afr J Surg. 2016;54(2):28-34.

38. van Schuppen J, Olthof DC, Wilde JC, Beenen LF, van Rijn RR, Goslings JC. Diagnostic accuracy of a step-up imaging strategy in pediatric patients with blunt abdominal trauma. Eur J Radiol. 2014;83(1):206-211.

39. Favot M, Courage C, Mantouffel J, Amponsah D. Ultrasound training in the emergency medicine clerkship. West J Emerg Med. 2015;16(6): 938-942.

40. Connolly K, Beier L, Langdorf MI, Anderson CL, Fox JC. Ultrafest: a novel approach to ultrasound in medical education leads to improvement in written and clinical examinations. West J Emerg Med. 2015;16(1):143-148.

41. Blackstock U, Munson J, Szyld D. Bedside ultrasound curriculum for medical students: report of a blended learning curriculum implementation and validation. J Clin Ultrasound. 2015;43(3):139-144.

42. Shokoohi H, Boniface K, Kaviany P, Armstrong P, Calabrese K, Pourmand A. An experiential learning model facilitates learning of bedside ultrasound by preclinical medical students. J Surg Educ. 2016;73(2):208-214.

43. Bowra J, Forrest-Horder S, Caldwell E, Cox M, D'Amours SK. Validation of nurse-performed FAST ultrasound. Injury. 2010;41(5):484-487.

44. Stolz LA, Muruganandan KM, Bisanzo MC, et al. Point-of-care ultrasound education for non-physician clinicians in a resource-limited emergency department. Trop Med Int Health. 2015;20(8):1067-1072.

45. Crouch AK, Dawson M, Long D, Allred D, Madsen T. Perceived confidence in the FAST exam before and after an educational intervention in a developing country. Int J Emerg Med. 2010;3(1):49-52.

46. Ziesmann MT, Park J, Unger BJ, et al. Validation of the quality of ultrasound imaging and competence (QUICk) score as an objective assessment tool for the FAST examination. J Trauma Acute Care Surg. 2015;78(5):1008-1013.

47. Platz E, Goldflam K, Mennicke M, Parisini E, Christ M, Hohenstein C. Comparison of Web-versus classroom-based basic ultrasonographic and EFAST training in 2 European hospitals. Ann Emerg Med. 2010;56(6):660-667.

48. Platz E, Liteplo A, Hurwitz S, Hwang J. Are live instructors replaceable? Computer vs. classroom lectures for EFAST training. J Emerg Med. 2011;40(5):534-538.

49. Bentley S, Mudan G, Strother C, Wong N. Are live ultrasound models replaceable? Traditional versus simulated education module for FAST exam. West J Emerg Med. 2015;16(6):818-822.
50. Damewood S, Jeanmonod D, Cadigan B. Comparison of a multimedia simulator to a human model for teaching FAST exam image interpretation and image acquisition. Acad Emerg Med. 2011;18(4):413-419.

51. Paddock MT, Bailitz J, Horowitz R, Khishfe B, Cosby K, Sergel MJ. Disaster response team FAST skills training with a portable ultrasound simulator compared to traditional training: pilot study. West J Emerg Med. 2015;16(2):325-330.

52. Lobo V, Hunter-Behrend M, Cullnan E, et al. Caudal edge of the liver in the Right Upper Quadrant (RUQ) view is the most sensitive area for free fluid on the FAST exam. West J Emerg Med. 2017;18(2): 270-280.

53. Jones R, Tabbut M, Gramer D. Elongated left lobe of the liver mimicking a subcapsular hematoma of the spleen on the focused assessment with sonography for trauma exam. Am J Emerg Med. 2014;32(7):814 e3-e4.

54. Sierzenski PR, Schofer JM, Bauman MJ, Nomura JT. The doubleline sign: a false positive finding on the Focused Assessment with Sonography for Trauma (FAST) examination. J Emerg Med. 2011;40(2):188-189.

55. Patwa AS, Cipot S, Lomibao A, et al. Prevalence of the "double-line" sign when performing focused assessment with sonography in trauma (FAST) examinations. Intern Emerg Med. 2015;10(6):721-724.

56. O’Brien KM, Stolz LA, Amini R, Gross A, Stolz U, Adhikari S. Focused assessment With sonography for trauma examination: reexamining the importance of the left upper quadrant view. $J$ Ultrasound Med. 2015;34(8):1429-1434.

57. Schnuriger B, Kilz J, Inderbitzin D, et al. The accuracy of FAST in relation to grade of solid organ injuries: a retrospective analysis of 226 trauma patients with liver or splenic lesion. BMC Med Imaging. 2009;9:3.

58. Von Kuenssberg Jehle D, Stiller G, Wagner D. Sensitivity in detecting free intraperitoneal fluid with the pelvic views of the FAST exam. Am J Emerg Med. 2003;21(6):476-478.

59. Laselle BT, Byyny RL, Haukoos JS, et al. False-negative FAST examination: associations with injury characteristics and patient outcomes. Ann Emerg Med. 2012;60(3):326-334.e3.

60. Rozycki GS, Knudson MM, Shackford SR, Dicker R. Surgeonperformed bedside organ assessment with sonography after trauma (BOAST): a pilot study from the WTA Multicenter Group. J Trauma. 2005;59(6):1356-1364.

61. Jang T, Kryder G, Sineff S, Naunheim R, Aubin C, Kaji AH. The technical errors of physicians learning to perform focused assessment with sonography in trauma. Acad Emerg Med. 2012;19(1):98-101.

62. Sauter TC, Hoess S, Lehmann B, Exadaktylos AK, Haider DG. Detection of pneumothoraces in patients with multiple blunt trauma: use and limitations of eFAST. Emerg Med J. 2017.
Open Access Emergency Medicine

\section{Publish your work in this journal}

The Open Access Emergency Medicine is an international, peerreviewed, open access journal publishing original research, reports, editorials, reviews and commentaries on all aspects of emergency medicine. The manuscript management system is completely online and includes a very quick and fair peer-review system, which is all

\section{Dovepress}

easy to use. Visit http://www.dovepress.com/testimonials.php to read real quotes from published authors. 